\title{
Fracture Mechanics for a Mode III Crack in a Magnetoelectroelastic Solid
}

\author{
Cun-Fa Gao, Pin Tong and Tong-Yi Zhang* \\ Department of Mechanical Engineering, Hong Kong University of Science and Technology \\ Clear Water Bay, Kowloon, Hong Kong, China
}

\begin{abstract}
In this paper, we developed a Stroh-type formalism for anti-plane deformation and then investigated the fracture mechanics for an elliptical cavity in a magnetoelectroelastic solid under remotely uniform in-plane electromagnetic and/or anti-plane mechanical loading, which allowed us to take the electromagnetic field inside the cavity into account. Reducing the cavity into a crack, we had explicit solutions in closed forms for a mode III crack, which included the extreme cases for an impermeable crack and a permeable crack. The results were illustrated with plots, showing that in the absence of mechanical loads, an applied electric or magnetic field, positive or negative, always tended to close the crack. On the other hand, in the presence of a mechanical load, a negative electric or magnetic field retarded crack growth, while a positive field could either enhance or retard crack propagation, depending on the strengths of the applied electric/magnetic fields and the level of the mechanical load as well. In other words, the effect of electric/magnetic fields on the fracture behavior is mechanical load-dependent.
\end{abstract}

Keywords: crack, magnetoelectroelastic solid, electric-magnetic field, energy release rate

\footnotetext{
* Corresponding author, Tel. +852-2358-7193, Fax +852-2358-1543, E-mail: mezhangt@ust.hk
} 


\section{Introduction}

Composite materials consisting of a piezoelectric phase and a piezomagnetic phase simultaneously process piezoelectric, piezomagnetic and magneto-electric effects, and thus they have wide applications in microwave electronics, optoelectronics and electronic instrumentation (Van Run et al., 1974). Duo to multi-field-coupled effects, a magnetic field may induce an electric field and an elastic field in a magneto-electro-elastic solid, and vise versa. The coupled properties of piezoelectric-piezomagnetic composites offer great opportunities for engineers to create intelligent structures and devices that are capable of responding to internal and/or environment changes. However, defects are often unavoidable in such materials and affect the performance and reliability of the end products. Thus, in recent years, the study of magnetoelectroelastic materials with defects has received considerable interests. Chung and Ting (1995) addressed two-dimensional (2D) Green functions for a magnetoelectroelastic anisotropic medium with an elliptical cavity or rigid inclusion. Pan (2002) obtained the three-dimensional Green functions in anisotropic magneto-electro-elastic bimaterials. Liu et al. (2001) derived Green's functions for an elliptical cavity by taking into account the electric-magnetic fields inside the cavity. They also gave the intensity factors associated with cracks in a closed form. Wang and Shen (2002) presented a general solution of threedimensional (3D) problems in magnetoelectroelastic media. Pan (2002) derived the 3D Green's functions in anisotropic magneto-electro-elastic bimaterails. Gao et al. (2003a,b) studied the single and collinear permeable crack problems using an elliptical-cavity-based approach and a crack-based method, respectively. They presented the explicit solutions for the electric/magnetic fields both inside and outside the cracks, and for the field intensity factors. Recently, Wang and Mai (2003) gave closed-form expressions for the energy release rate of an impermeable or permeable crack in a 
piezomagnetic/piezoelectric solid. However, it should be noted that all these works for crack problems were made based on the mathematical crack assumption that a crack was a slit with a zero width and thus did not take the effect of initial crack width or crack opening into account. As a result, it is shown from the available solutions for a permeable mathematical crack that electricmagnetic loading has no influence on the crack growth. A real crack, however, has a finite nonzero width. Zhang (1994), Zhang and Tong (1996) and Zhang et al. (1998) studied the crack width effect for piezoelectric materials, showing that the crack width has great influence on the crack growth because of the existence of electric field inside the crack. In the present work we shall explore the effects of electric-magnetic fields on the fracture behavior of a mode III crack in a magnetoelectroelastic solid under mechanical, electrical and/or magnetic loading. In addition, we shall develop a methodology, a Stroh-type formalism for anti-plane deformation of magnetoelectroelastic materials.

This work is organized as follows: Section 2 gives basic governing equations for a linear magnetoelectroelastic solid and the Stroh-type formalism for anti-plane deformation. This formalism allows one to extend easily the present results to complicated generalized 2D cases. Electric/magnetic fields inside the crack and the field intensity factors are derived in Section 3. Section 4 presents an explicit solution for the energy release rate. The corresponding results for permeable and impermeable cracks are also given as special cases. In Section 5, numerical results are presented to graphically show the effects of electric/magnetic fields on the energy release rate. Finally, Section 6 concludes the present work. 


\section{Basic equations}

For a linear magnetoelectroelastic solid in a rectangular coordinate system, $x_{i}(i=1,2,3)$, in the absence of body forces, electric charge density and electric current, the complete set of the basic equations is

$$
\begin{gathered}
\sigma_{i j}=C_{i j k l} \varepsilon_{k l}-e_{k i j} E_{k}-q_{k i j} H_{k}, \\
D_{i}=e_{i k l} \varepsilon_{k l}+\kappa_{i k} E_{k}+d_{i k} H_{k}, \\
B_{i}=q_{i k l} \varepsilon_{k l}+d_{i k} E_{k}+\mu_{i k} H_{k}, \\
\sigma_{i j, j}=0, D_{i, i}=0, B_{i, i}=0, \\
\varepsilon_{i j}=\frac{1}{2}\left(u_{i, j}+u_{j, i}\right), \\
E_{i}=-\varphi_{, i}, \\
H_{i}=-\psi(,
\end{gathered}
$$

where a comma in the subscripts stands for partial differentiation; repeated indices denote summation; $\sigma_{i j}, \varepsilon_{i j}, E_{i}, D_{i}, H_{i}$ and $B_{i}$ are the stress, strain, electric field, electric displacement, magnetic field and magnetic induction, respectively; $u_{k}(k=1,2,3), \varphi$ and $\psi$ stand for the displacement, the electric potential and the magnetic potential, respectively; $e_{k i j}, q_{k i j}$ and $d_{i k}$ are the piezoelectric, piezomagnetic and magnetoelectric coupling tensors, respectively; $C_{i j k l}, \kappa_{i k}$ and $\mu_{i l}$ are the elastic stiffness tensor, the dielectric permittivities and the magnetic permeabilities, respectively. The material constants have the following symmetries: 


$$
C_{i j k l}=C_{j i k l}=C_{i j l k}=C_{k l i j}, e_{k i j}=e_{k j i}, q_{k i j}=q_{k j i}, \kappa_{k l}=\kappa_{l k}, d_{k l}=d_{l k}, \mu_{k l}=\mu_{l k} .
$$

Moreover, $C_{i j k l}, \kappa_{k l}$ and $\mu_{k l}$ are positive definite in the sense that

$$
C_{i j k l} \varepsilon_{i j} \varepsilon_{k l}>0, \kappa_{k l} E_{k} E_{l}>0, \mu_{k l} H_{k} H_{l}>0
$$

for non-zero $\varepsilon_{i j}, E_{k}$ and $H_{k}$. For a transversely isotropic magnetoelectroelastic solid with the $x_{3}$ axis being the symmetry axis, the constitutive equation (1) can be written in the Voigt form as

$$
\left\{\begin{array}{l}
\sigma_{11} \\
\sigma_{22} \\
\sigma_{33} \\
\sigma_{32} \\
\sigma_{31} \\
\sigma_{12}
\end{array}\right\}=\left[\begin{array}{cccccc}
c_{11} & c_{12} & c_{13} & 0 & 0 & 0 \\
c_{12} & c_{11} & c_{13} & 0 & 0 & 0 \\
c_{13} & c_{13} & c_{33} & 0 & 0 & 0 \\
0 & 0 & 0 & c_{44} & 0 & 0 \\
0 & 0 & 0 & 0 & c_{44} & 0 \\
0 & 0 & 0 & 0 & 0 & \left(c_{11}-c_{12}\right) / 2
\end{array}\right]\left\{\begin{array}{c}
\varepsilon_{11} \\
\varepsilon_{22} \\
\varepsilon_{33} \\
2 \varepsilon_{32} \\
2 \varepsilon_{31} \\
2 \varepsilon_{12}
\end{array}\right\}
$$

$$
\begin{gathered}
-\left[\begin{array}{ccc}
0 & 0 & e_{31} \\
0 & 0 & e_{31} \\
0 & 0 & e_{33} \\
0 & e_{15} & 0 \\
e_{15} & 0 & 0 \\
0 & 0 & 0
\end{array}\right]\left\{\begin{array}{l}
E_{1} \\
E_{2} \\
E_{3}
\end{array}\right\}-\left[\begin{array}{ccc}
0 & 0 & q_{31} \\
0 & 0 & q_{31} \\
0 & 0 & q_{33} \\
0 & q_{15} & 0 \\
q_{15} & 0 & 0 \\
0 & 0 & 0
\end{array}\right]\left\{\begin{array}{l}
H_{1} \\
H_{2} \\
H_{3}
\end{array}\right\}, \quad(4) \\
\left\{\begin{array}{l}
D_{1} \\
D_{2} \\
D_{3}
\end{array}\right\}=\left[\begin{array}{cccccc}
0 & 0 & 0 & 0 & e_{15} & 0 \\
0 & 0 & 0 & e_{15} & 0 & 0 \\
e_{31} & e_{31} & e_{33} & 0 & 0 & 0
\end{array}\right]\left\{\begin{array}{c}
\varepsilon_{11} \\
\varepsilon_{22} \\
\varepsilon_{33} \\
2 \varepsilon_{32} \\
2 \varepsilon_{31} \\
2 \varepsilon_{12}
\end{array}\right\}+\left[\begin{array}{ccc}
\kappa_{11} & 0 & 0 \\
0 & \kappa_{22} & 0 \\
0 & 0 & \kappa_{33}
\end{array}\right]\left\{\begin{array}{l}
E_{1} \\
E_{2} \\
E_{3}
\end{array}\right\}+\left[\begin{array}{ccc}
d_{11} & 0 & 0 \\
0 & d_{22} & 0 \\
0 & 0 & d_{33}
\end{array}\right]\left\{\begin{array}{l}
H_{1} \\
H_{2} \\
H_{3}
\end{array}\right\},
\end{gathered}
$$




$$
\left\{\begin{array}{l}
B_{1} \\
B_{2} \\
B_{3}
\end{array}\right\}=\left[\begin{array}{cccccc}
0 & 0 & 0 & 0 & q_{15} & 0 \\
0 & 0 & 0 & q_{15} & 0 & 0 \\
q_{31} & q_{31} & q_{33} & 0 & 0 & 0
\end{array}\right]\left\{\begin{array}{l}
\varepsilon_{11} \\
\varepsilon_{22} \\
\varepsilon_{33} \\
2 \varepsilon_{32} \\
2 \varepsilon_{31} \\
2 \varepsilon_{12}
\end{array}\right\}+\left[\begin{array}{ccc}
d_{11} & 0 & 0 \\
0 & d_{22} & 0 \\
0 & 0 & d_{33}
\end{array}\right]\left\{\begin{array}{l}
E_{1} \\
E_{2} \\
E_{3}
\end{array}\right\}+\left[\begin{array}{ccc}
\mu_{11} & 0 & 0 \\
0 & \mu_{22} & 0 \\
0 & 0 & \mu_{33}
\end{array}\right]\left\{\begin{array}{l}
H_{1} \\
H_{2} \\
H_{3}
\end{array}\right\} .
$$

Since the anti-plane solution is relatively simple and can be written in an explicit form, thereby clearly providing physical insights into the fracture behavior of magnetoelectroelastic materials, we shall study a mode III crack in the isotropic plane in the present work. For the two-dimensional antiplane deformation, the out-of-plane displacement and the in-plane electric/magnetic fields are functions of $x_{1}$ and $x_{2}$ only, that

$$
u_{1}=u_{2}=0, u_{3}=u_{3}\left(x_{1}, x_{2}\right), \varphi=\varphi\left(x_{1}, x_{2}\right), \psi=\psi\left(x_{1}, x_{2}\right) .
$$

In this case, we have by using (3)-(7) that

$$
\begin{aligned}
& \sigma_{3 k}=c_{44} \frac{\partial u_{3}}{\partial x_{k}}+e_{15} \frac{\partial \varphi}{\partial x_{k}}+q_{15} \frac{\partial \psi}{\partial x_{k}}, \\
& D_{k}=e_{15} \frac{\partial u_{3}}{\partial x_{k}}-\kappa_{11} \frac{\partial \varphi}{\partial x_{k}}-d_{11} \frac{\partial \psi}{\partial x_{k}}, \\
& B_{k}=q_{15} \frac{\partial u_{3}}{\partial x_{k}}-d_{11} \frac{\partial \varphi}{\partial x_{k}}-\mu_{11} \frac{\partial \psi}{\partial x_{k}} \quad(k=1,2) .
\end{aligned}
$$

Furthermore, substituting Eqs. (8)-(10) into Eq. (2) results in

$$
\mathbf{B}_{0} \nabla^{2} \mathbf{u}=\mathbf{0},
$$

where $\nabla^{2}=\partial^{2} / \partial x_{1}^{2}+\partial^{2} / \partial x_{2}^{2}$, and

$$
\mathbf{u}=\left[u_{3}, \varphi, \psi\right]^{T},
$$

is called the generalized displacement vector, and 


$$
\mathbf{B}_{0}=\left[\begin{array}{ccc}
c_{44} & e_{15} & q_{15} \\
e_{15} & -\kappa_{11} & -d_{11} \\
q_{15} & -d_{11} & -\mu_{11}
\end{array}\right] .
$$

Since $\mathbf{B}_{0}$ is non-singular, from Eq. (11), we have

$$
\nabla^{2} \mathbf{u}=\mathbf{0}
$$

The general solution of Eq. (14) is simply

$$
\mathbf{u}=\mathbf{f}(z)+\overline{\mathbf{f}(z)}, \quad z=x_{1}+i x_{2},
$$

where $\mathbf{f}(z)$ is an analytic function.

To develop a Stroh-type formalism for anti-plane deformation, we introduce a generalized stress function vector, $\phi$, such that

$$
\boldsymbol{\Sigma}_{1}=\left[\sigma_{31}, D_{1}, B_{1}\right]^{T}=-\phi_{, 2}, \boldsymbol{\Sigma}_{2}=\left[\sigma_{32}, D_{2}, B_{2}\right]^{T}=\phi_{, 1} .
$$

Substituting Eqs. (8)-(10) into Eq. (16) yields

$$
\begin{aligned}
-\phi_{, 2} & =\mathbf{B}_{0} \frac{\partial \mathbf{f}}{\partial x_{1}}+\mathbf{B}_{0} \frac{\overline{\partial \mathbf{f}}}{\partial x_{1}}, \\
\phi_{, 1} & =\mathbf{B}_{0} \frac{\partial \mathbf{f}}{\partial x_{2}}+\mathbf{B}_{0} \frac{\overline{\partial \mathbf{f}}}{\partial x_{2}} .
\end{aligned}
$$

Thus, we obtain from (17) or (18) that

$$
\phi=i \mathbf{B}_{0} \mathbf{f}(z)-i \mathbf{B}_{0} \overline{\mathbf{f}(z)} .
$$

To let Eqs. (15) and (19) appear like those in the Stroh formalism, we rewrite them as

$$
\begin{gathered}
\mathbf{u}=\mathbf{A f f}(z)+\overline{\mathbf{A f}(z),} \\
\phi=\mathbf{B f}(z)+\overline{\mathbf{B}} \overline{\mathbf{f}(z)}, z=x_{1}+i x_{2},
\end{gathered}
$$

where 


$$
\mathbf{A}=\mathbf{I}, \mathbf{B}=i \mathbf{B}_{0}
$$

Equations (20) and (21) can be written also as

$$
\begin{aligned}
& \phi=2 \operatorname{Re}[\mathbf{k}(z)], \\
& \mathbf{u}=2 \operatorname{Im}[\mathbf{Y k}(z)],
\end{aligned}
$$

where

$$
\begin{aligned}
& \mathbf{k}(z)=\mathbf{B f}(z), \\
& \mathbf{Y}=i \mathbf{A B}^{-1}
\end{aligned}
$$

From Eq. (22) and Eq. (25b), we find

$$
\mathbf{Y}=\mathbf{B}_{0}^{-1}
$$

Let us define

$$
\mathbf{H}=2 \operatorname{Re}[\mathbf{Y}]
$$

Since $\mathbf{Y}$ is real in the present case, Eq. (27) is simplified to

$$
\mathbf{H}=2 \mathbf{Y} .
$$

Using Eq. (13) and Eq. (26), we have

$$
\mathbf{H}=2 \mathbf{Y}=\frac{2}{\nabla}\left[\begin{array}{ccc}
\kappa_{11} \mu_{11}-d_{11}^{2} & e_{15} \mu_{11}-d_{11} q_{15} & \kappa_{11} q_{15}-d_{11} e_{15} \\
e_{15} \mu_{11}-d_{11} q_{15} & -q_{15}^{2}-c_{44} \mu_{11} & c_{44} d_{11}+e_{15} q_{15} \\
\kappa_{11} q_{15}-d_{11} e_{15} & c_{44} d_{11}+e_{15} q_{15} & -e_{15}^{2}-c_{44} d_{11}^{2}
\end{array}\right],
$$

where

$$
\nabla=c_{44} \kappa_{11} \mu_{11}+e_{15}^{2} \mu_{11}+\kappa_{11} q_{15}^{2}-2 d_{11} e_{15} q_{15}-c_{44} d_{11}^{2}
$$

The above derivations indicate that we will have the stress, electric, and magnetic fields if the complex potential $\mathbf{f}(z)$ is available. The complex potential vector, $\mathbf{f}(z)$, is determined from 
boundary conditions. All the deviations described above like those in the Stroh formalism except that the eigen-roots are all " $i=\sqrt{-1}$ " in the present study of anti-deformation.

\section{Electric-magnetic field inside the crack and field intensity factors}

Consider an elliptical cavity in a transversely isotropic magnetoelectroelastic solid infinitely large. The cavity is filled with air of a dielectric permittivity, $\kappa_{0}$, and a magnetic permeability, $\mu_{0}$.

It is assumed that the electric and magnetic poling directions are both along the positive $x_{3}$ axis and the isotropic plane is in the $x_{1}-x_{2}$ plane. The solid is under uniform remote anti-plane shear and inplane electric-magnetic field loadings, $\boldsymbol{\Sigma}_{2 \infty}=\left[\sigma_{32}^{\infty}, D_{2}^{\infty}, B_{2}^{\infty}\right]^{T}$ and $\boldsymbol{\Sigma}_{1 \infty}=\left[\sigma_{31}^{\infty}, D_{1}^{\infty}, B_{1}^{\infty}\right]^{T}$, as shown in Fig. 1. In the following analysis, we assume that the cavity surface is free of traction, external charge and electric current.

Inside the cavity, the electric field is uniform and the potential functions can be expressed as

$$
\begin{aligned}
& \phi_{0}=\left(D_{2}^{0} x_{1}-D_{1}^{0} x_{2}\right) \mathbf{i}_{2}+\left(B_{2}^{0} x_{1}-B_{1}^{0} x_{2}\right) \mathbf{i}_{3}, \\
& \varphi_{0}=-E_{1}^{0} x_{1}-E_{2}^{0} x_{2}, \\
& \psi_{0}=-H_{1}^{0} x_{1}-H_{2}^{0} x_{2},
\end{aligned}
$$

where $\mathbf{i}_{2}=[0,1,0]^{T}, \mathbf{i}_{3}=[0,0,1]^{T}$, and $D_{k}^{0}, B_{k}^{0}, H_{k}^{0}$ and $E_{k}^{0}$ are the components of the electric and magnetic fields, respectively, which are constant and will be determined by the loading condition. In the solid, the complex function $\mathbf{f}(z)$ takes the form:

$$
\mathbf{f}(\mathrm{z})=\mathbf{c}^{\infty} z+\mathbf{f}_{0}(z)
$$


where $\mathbf{c}^{\infty}$ is a constant related to the loading condition at infinity, and $\mathbf{f}_{0}(z)$ is an unknown complex function, which vanishes at infinity, i.e., $\mathbf{f}_{0}(\infty)=\mathbf{0}$. Substituting Eq. (33) into Eqs. (23) and (24) and noting that $\mathbf{Y}$ is real, we have

$$
\begin{aligned}
& \boldsymbol{\phi}=\boldsymbol{\Sigma}_{2 \infty} x_{1}-\boldsymbol{\Sigma}_{1 \infty} x_{2}+2 \operatorname{Re}\left[\mathbf{k}_{0}(z)\right], \\
& \varphi=-E_{1}^{\infty} x_{1}-E_{2}^{\infty} x_{2}+2 \operatorname{Im}\left[\mathbf{Y k}_{0}(z)\right]_{2}, \\
& \varphi=-H_{1}^{\infty} x_{1}-H_{2}^{\infty} x_{2}+2 \operatorname{Im}\left[\mathbf{Y k}_{0}(z)\right]_{3},
\end{aligned}
$$

where []$_{J}$ stands for the $J^{\text {th }}$ row of the vector [] and $\mathbf{k}_{0}(z)=\mathbf{B f}_{0}(z)$.

On the cavity surface, the continuity of the appropriate field variables requires

$$
\phi_{0}=\phi, \varphi=\varphi_{0}, \psi=\psi_{0} .
$$

Let us introduce the transformation function,

$$
z(\zeta)=R\left(\zeta+m \zeta^{-1}\right) \text { with } R=\frac{a+b}{2} \text { and } m=\frac{a-b}{a+b},
$$

which maps the elliptical cavity in the z-plane into a unit circle in the $\zeta$-plane. On the cavity surface, one has $\zeta=\sigma=e^{i \theta}$. Then, Eq. (37) becomes

$$
\phi_{0}(\sigma)=\phi(\sigma), \varphi(\sigma)=\varphi_{0}(\sigma), \psi(\sigma)=\psi_{0}(\sigma) .
$$

Substituting Eqs. (30)-(32) and Eqs. (34)-(36) together with

$$
\begin{aligned}
& x_{1}=a \cos \theta=\frac{1}{2} a\left(\frac{1}{\sigma}+\sigma\right), \\
& x_{2}=b \sin \theta=\frac{1}{2} i b\left(\frac{1}{\sigma}-\sigma\right),
\end{aligned}
$$

into Eq. (38), we obtain 


$$
\mathbf{k}_{0}(z)=-\frac{1}{2} \zeta^{-1}(z) \mathbf{p}
$$

where

$$
\begin{gathered}
\zeta^{-1}(z)=\frac{z-\sqrt{z^{2}-a^{2}+b^{2}}}{a-b}, \\
\mathbf{p}=\left[a \sigma_{32}^{\infty}-i b \sigma_{31}^{\infty}, a \Delta D_{2}^{\infty}-i b \Delta D_{1}^{\infty}, a \Delta B_{2}^{\infty}-i b \Delta B_{1}^{\infty}\right]^{T}, \\
\Delta D_{k}^{\infty}=D_{k}^{\infty}-D_{k}^{0}, \Delta B_{k}^{\infty}=B_{k}^{\infty}-B_{k}^{0},(k=1,2) .
\end{gathered}
$$

The four unknown $D_{k}^{0}$ and $B_{k}^{0}$ in Eq. (42) can be determined from the following four equations:

$$
\begin{aligned}
& \operatorname{Re}\left[Y_{21} \mathbf{M}^{\infty}\right]+a \operatorname{Re}\left[Y_{22}\right] \Delta D_{2}^{\infty}+a \operatorname{Re}\left[Y_{23}\right] \Delta B_{2}^{\infty}=b\left(E_{2}^{\infty}-E_{2}^{0}\right), \\
& \operatorname{Re}\left[Y_{31} \mathbf{M}^{\infty}\right]+a \operatorname{Re}\left[Y_{32}\right] \Delta D_{2}^{\infty}+a \operatorname{Re}\left[Y_{33}\right] \Delta B_{2}^{\infty}=b\left(H_{2}^{\infty}-H_{2}^{0}\right), \\
& \operatorname{Im}\left[Y_{21} \mathbf{M}^{\infty}\right]-b \operatorname{Re}\left[Y_{22}\right] \Delta D_{1}^{\infty}-b \operatorname{Re}\left[Y_{23}\right] \Delta B_{1}^{\infty}=-a\left(E_{1}^{\infty}-E_{1}^{0}\right), \\
& \operatorname{Im}\left[Y_{31} \mathbf{M}^{\infty}\right]-b \operatorname{Re}\left[Y_{32}\right] \Delta D_{1}^{\infty}-b \operatorname{Re}\left[Y_{33}\right] \Delta B_{1}^{\infty}=-a\left(H_{1}^{\infty}-H_{1}^{0}\right),
\end{aligned}
$$

where

$$
\mathbf{M}^{\infty}=\left[a \sigma_{32}^{\infty}-i b \sigma_{31}^{\infty}\right]
$$

Since $\mathbf{Y}$ is real and $2 \mathrm{Re}[\mathbf{Y}]=\mathbf{H}$ in the present case, Eqs. (43)-(46) can be reduced further to

$$
\begin{gathered}
H_{21} \sigma_{32}^{\infty}+H_{22} \Delta D_{2}^{\infty}+H_{23} \Delta B_{2}^{\infty}=2 \alpha\left(E_{2}^{\infty}-E_{2}^{0}\right), \\
H_{31} \sigma_{32}^{\infty}+H_{32} \Delta D_{2}^{\infty}+H_{33} \Delta B_{2}^{\infty}=2 \alpha\left(H_{2}^{\infty}-H_{2}^{0}\right), \\
\alpha\left(H_{21} \sigma_{31}^{\infty}+H_{22} \Delta D_{1}^{\infty}+H_{23} \Delta B_{1}^{\infty}\right)=2\left(E_{1}^{\infty}-E_{1}^{0}\right),
\end{gathered}
$$




$$
\alpha\left(H_{31} \sigma_{31}^{\infty}+H_{32} \Delta D_{1}^{\infty}+H_{33} \Delta B_{1}^{\infty}\right)=2\left(H_{1}^{\infty}-H_{1}^{0}\right)
$$

where $\alpha=b / a$.

Using the relationships $D_{2}^{0}=\kappa_{0} E_{2}^{0}, B_{2}^{0}=\mu_{0} H_{2}^{0}$ and Eq. (42), we find

$$
E_{2}^{\infty}-E_{2}^{0}=E_{2}^{\infty}+\frac{\Delta D_{2}^{\infty}-D_{2}^{\infty}}{\kappa_{0}}, H_{2}^{\infty}-H_{2}^{0}=H_{2}^{\infty}+\frac{\Delta B_{2}^{\infty}-B_{2}^{\infty}}{\mu_{0}}
$$

Substituting Eq. (52) into Eqs. (48) and (49) leads to

$$
\begin{aligned}
& H_{21} \sigma_{32}^{\infty}+\left(H_{22}-\frac{2 \alpha}{\kappa_{0}}\right) \Delta D_{2}^{\infty}+H_{23} \Delta B_{2}^{\infty}=2 \alpha E_{2}^{\infty}-\frac{2 \alpha}{\kappa_{0}} D_{2}^{\infty} \\
& H_{31} \sigma_{32}^{\infty}+H_{32} \Delta D_{2}^{\infty}+\left(H_{33}-\frac{2 \alpha}{\mu_{0}}\right) \Delta B_{2}^{\infty}=2 \alpha H_{2}^{\infty}-\frac{2 \alpha}{\mu_{0}} B_{2}^{\infty} .
\end{aligned}
$$

Define two parameters $\lambda_{e}$ and $\lambda_{m}$ as

$$
\lambda_{e}=\frac{\alpha}{\beta_{e}}, \lambda_{m}=\frac{\alpha}{\beta_{m}}
$$

in which

$$
\beta_{e}=\kappa_{0} / \kappa^{e f f}, \beta_{m}=\mu_{0} / \mu^{e f f}, \kappa^{e f f}=-1 / Y_{22}, \mu^{e f f}=-1 / Y_{33},
$$

where $\kappa^{\text {eff }}$ and $\mu^{\text {eff }}$ are the effective dielectric permittivity and magnetic permeability of the material, respectively. The two parameters $\lambda_{e}$ and $\lambda_{m}$ characterize the permeability of the crack to electric/magnetic fields. The crack is impermeable if $\lambda_{e} \rightarrow \infty$ and $\lambda_{m} \rightarrow \infty$, permeable if $\lambda_{e}=\lambda_{m}=0$, and semi-permeable if $\lambda_{e}$ and $\lambda_{m}$ are nonzero finite constants. A parameter similar to $\lambda_{e}$ was introduced by McMeeking (1989) and Zhang et al. $(1996,1998)$ in their studies of the fracture of dielectric and piezoelectric materials, respectively. Note that $\lambda_{e}$ and $\lambda_{m}$ are mutually 
dependent parameters with $\frac{\lambda_{m}}{\lambda_{e}}=\frac{\kappa_{0}}{\mu_{0}} \frac{Y_{22}}{Y_{33}}$ being constant for a given material. This means that if a crack is electrically permeable (impermeable), the crack is also magnetically permeable (impermeable). Using Eq. (55), we can rewrite Eqs. (53) and (54) as

$$
\begin{aligned}
& H_{21} \sigma_{32}^{\infty}+H_{22}\left(1+\lambda_{e}\right) \Delta D_{2}^{\infty}+H_{23} \Delta B_{2}^{\infty}=2 \alpha E_{2}^{\infty}+\lambda_{e} H_{22} D_{2}^{\infty}, \\
& H_{31} \sigma_{32}^{\infty}+H_{32} \Delta D_{2}^{\infty}+H_{33}\left(1+\lambda_{m}\right) \Delta B_{2}^{\infty}=2 \alpha H_{2}^{\infty}+\lambda_{m} H_{33} B_{2}^{\infty} .
\end{aligned}
$$

For a semi-permeable crack with $\alpha \rightarrow 0$, while $\lambda_{e}$ and $\lambda_{m}$ being kept finite, neglecting $\alpha$ in Eqs. (50), (51), (56) and (57) results in

$$
\begin{aligned}
& E_{1}^{0}=E_{1}^{\infty}, H_{1}^{0}=H_{1}^{\infty}, \\
& H_{22}\left(1+\lambda_{e}\right) \Delta D_{2}^{\infty}+H_{23} \Delta B_{2}^{\infty}=\lambda_{e} H_{22} D_{2}^{\infty}-H_{21} \sigma_{32}^{\infty}, \\
& H_{32} \Delta D_{2}^{\infty}+H_{33}\left(1+\lambda_{m}\right) \Delta B_{2}^{\infty}=\lambda_{m} H_{33} B_{2}^{\infty}-H_{31} \sigma_{32}^{\infty} .
\end{aligned}
$$

Solving Eqs. (59) and (60) gives

$$
\begin{aligned}
\Delta D_{2}^{\infty} & =\frac{1}{\eta}\left\{\left[H_{23} H_{31}-\left(1+\lambda_{m}\right) H_{21} H_{33}\right] \sigma_{32}^{\infty}+\lambda_{e}\left(1+\lambda_{m}\right) H_{33} H_{22} D_{2}^{\infty}-\lambda_{m} H_{23} H_{33} B_{2}^{\infty}\right\}, \\
\Delta B_{2}^{\infty} & =\frac{1}{\eta}\left\{\left[H_{32} H_{21}-\left(1+\lambda_{e}\right) H_{31} H_{22}\right] \sigma_{32}^{\infty}+\lambda_{m}\left(1+\lambda_{e}\right) H_{22} H_{33} B_{2}^{\infty}-\lambda_{e} H_{32} H_{22} D_{2}^{\infty}\right\},
\end{aligned}
$$

where

$$
\eta=H_{22} H_{33}\left(1+\lambda_{e}\right)\left(1+\lambda_{m}\right)-H_{23}^{2} .
$$

We define the intensity factor vector as 


$$
\mathbf{k}=\left(k_{\sigma}, k_{D}, k_{B}\right)^{T}=\lim _{x_{1} \rightarrow a} \sqrt{2 \pi}\left(x_{1}-a\right)^{1 / 2} \phi_{, 1}\left(x_{1}\right) .
$$

Substituting Eq. (34) together with Eq. (39) into Eq. (63) results in

$$
\mathbf{k}=\sqrt{\pi a}\left(\sigma_{32}^{\infty}, \Delta D_{2}^{\infty}, \Delta B_{2}^{\infty}\right)^{T}
$$

in which $\Delta D_{2}^{\infty}$ and $\Delta B_{2}^{\infty}$ are determined from Eqs. (61) and (62). Equation (64) shows that the stress intensity factor relates to only the applied mechanical load, while the electric/magnetic intensity factors are dependent on mechanical and electric-magnetic loads, material constants as well as the values of $\lambda_{e}$ and $\lambda_{m}$, which are related to the crack opening.

\section{Energy Release Rate}

Zhang et al. (2002) discussed in detail that four thermodynamic potentials can be used to calculate the energy release rate for crack propagation in a piezoelectric material. The general energy balance for an infinitesimal crack extension can be written in the form

$$
\partial P=\partial W-\partial U
$$

where $W$ is the generalized work done by external mechanical and electrical-magnetic loads, $U$ is the generalized mechanical and electrical-magnetic energy stored inside the body of interest, and $\partial P$ denotes the change in the potential during creating the new crack surface. The generalized work and the generalized stored energy are given by

$$
\begin{aligned}
W & =\int_{\Gamma}\left(\sigma_{i j} n_{j} u_{i}+D_{i} n_{i} \varphi+B_{i} n_{i} \psi\right) d \Gamma, \\
U & =\iint_{\Omega} h d \Omega,
\end{aligned}
$$

respectively, where $\Gamma$ denotes a sufficiently large contour around the crack, $\Omega$ is the integration domain enclosed by $\Gamma, u_{i}$ is the mechanical displacement, $D_{i} n_{i}$ and $B_{i} n_{i}$ are the prescribed 
boundary values of the electric displacement and the magnetic induction, respectively, and $h$ is the electric-magnetic enthalpy per unit volume that

$$
h=\frac{1}{2} \sigma_{i j} \varepsilon_{i j}-\frac{1}{2} D_{i} E_{i}-\frac{1}{2} B_{i} H_{i} .
$$

The energy release rate, $\mathrm{G}$, is defined as

$$
G=\frac{1}{2} \frac{\partial P}{\partial a}
$$

where

$$
P=W-U
$$

Using Eqs. (70), and (66)-(68), we have

$$
P=-\frac{1}{2} \int_{\Gamma} d \phi^{\mathrm{T}} \cdot \mathbf{u}
$$

In the present work, we assume that the crack or cavity propagate in a self-similar fashion. Based on the assumption and some algebraic manipulations, we obtain, by using Eqs. (71), (20) and (21) that

$$
G=\frac{\pi a}{4} \Sigma_{2 \infty}^{T} \mathbf{H} \Pi_{2 \infty}
$$

where

$$
\Pi_{2 \infty}=\left\lfloor\sigma_{32}^{\infty}, D_{2}^{\infty}-D_{2}^{0}, B_{2}^{\infty}-B_{2}^{0}\right]^{T}
$$

Since $\Sigma_{2 \infty}=\Pi_{2 \infty}+\mathbf{i}_{2} D_{2}^{0}+\mathbf{i}_{3} B_{2}^{0}$, Eq. (72) can be rewritten as

$$
G=\frac{\pi a}{4} \Pi_{2 \infty}^{T} \mathbf{H} \Pi_{2 \infty}+\frac{\pi a}{4} \mathbf{i}_{2}^{T} \mathbf{H} \Pi_{2 \infty} D_{2}^{0}+\frac{\pi a}{4} \mathbf{i}_{3}^{T} \mathbf{H} \Pi_{2 \infty} B_{2}^{0},
$$

which can be further simplified as

$$
G=\frac{1}{4} \mathbf{k}^{T} \mathbf{H k}+\frac{\pi a}{4} \mathbf{i}_{2}^{T} \mathbf{H} \Pi_{2 \infty} D_{2}^{0}+\frac{\pi a}{4} \mathbf{i}_{3}^{T} \mathbf{H} \Pi_{2 \infty} B_{2}^{0},
$$


where $\mathbf{k}$ is the intensity factor vector defined by Eq. (64).

Equation (74) or (75) can be further simplified for special cases. For electrically and magnetically impermeable cracks, $D_{2}^{0}=B_{2}^{0}=0$, Eq. (74) becomes

$$
G=\frac{\pi a}{4}\left(\sigma_{32}^{\infty}, D_{2}^{\infty}, B_{2}^{\infty}\right) \mathbf{H}\left(\sigma_{32}^{\infty}, D_{2}^{\infty}, B_{2}^{\infty}\right)^{T},
$$

or

$$
G=\frac{1}{4} \mathbf{k}_{i m p}^{T} \mathbf{H} \mathbf{k}_{i m p},
$$

where $\mathbf{k}_{\text {imp }}$ is the intensity factor vector for impermeable cracks that

$$
\mathbf{k}_{i m p}=\sqrt{\pi a}\left(\sigma_{32}^{\infty}, D_{2}^{\infty}, B_{2}^{\infty}\right)^{T} .
$$

For a permeable crack, $\lambda_{e}=\lambda_{m}=0$, one has from Eqs. (61) and (62) that

$$
\begin{gathered}
\Delta D_{2}^{\infty}=D_{2}^{\infty}-D_{2}^{0}=-c_{D} \sigma_{32}^{\infty}, \\
\Delta B_{2}^{\infty}=B_{2}^{\infty}-B_{2}^{0}=-c_{B} \sigma_{32}^{\infty},
\end{gathered}
$$

where

$$
c_{D}=\frac{H_{21} H_{33}-H_{23} H_{31}}{H_{22} H_{33}-H_{23}^{2}}, c_{B}=\frac{H_{31} H_{22}-H_{32} H_{21}}{H_{22} H_{33}-H_{23}^{2}} .
$$

In the mean time, using Eq. (73), one obtains

$$
\mathbf{i}_{2}^{T} \mathbf{H} \Pi_{2 \infty}=H_{21} \sigma_{32}^{\infty}+H_{22}\left(D_{2}^{\infty}-D_{2}^{0}\right)+H_{23}\left(B_{2}^{\infty}-B_{2}^{0}\right) .
$$

Substituting Eqs. (79) and (80) into Eq.(81) leads to

$$
\mathbf{i}_{2}^{T} \mathbf{H} \Pi_{2 \infty}=0 .
$$

Similarly, we have 


$$
\mathbf{i}_{3}^{T} \mathbf{H} \Pi_{2 \infty}=0 .
$$

Substituting Eqs. (82) and (83) into Eq. (74) leads to

$$
G=\frac{\pi a}{4}\left(\sigma_{32}^{\infty}, D_{2}^{\infty}-D_{2}^{0}, B_{2}^{\infty}-B_{2}^{0}\right) \mathbf{H}\left(\sigma_{32}^{\infty}, D_{2}^{\infty}-D_{2}^{0}, B_{2}^{\infty}-B_{2}^{0}\right)^{T},
$$

that is

$$
G=\frac{1}{4} \mathbf{k}_{p}^{T} \mathbf{H} \mathbf{k}_{p},
$$

where $\mathbf{k}_{p}$ is the intensity factor vector for the permeable crack in the form of Eq. (64), in which the $\Delta D_{2}^{\infty}$ and $\Delta B_{2}^{\infty}$ are given by Eqs. (79) and (80). In summary, for a permeable crack, we have

$$
\begin{aligned}
& E_{1}^{0}=E_{1}^{\infty}, H_{1}^{0}=H_{1}^{\infty}, \\
& D_{2}^{0}=D_{2}^{\infty}+c_{D} \sigma_{32}^{\infty}, \\
& B_{2}^{0}=B_{2}^{\infty}+c_{B} \sigma_{32}^{\infty}, \\
& k_{\sigma}=\sqrt{\pi a} \sigma_{32}^{\infty}, \quad k_{D}=-c_{D} k_{\sigma}, \quad k_{B}=-c_{B} k_{\sigma}, \\
& G=\frac{\pi a}{4} H_{11}^{*}(0)\left(\sigma_{32}^{\infty}\right)^{2},
\end{aligned}
$$

where

$$
H_{11}^{*}(0)=H_{11}-\frac{H_{12}^{2} H_{33}-2 H_{12} H_{23} H_{31}+H_{13}^{2} H_{22}}{H_{22} H_{33}-H_{23}^{2}} .
$$

For a semi-permeable crack, $\lambda_{e}$ and $\lambda_{m}$ have finite nonzero values. In this case, Eq. (72) can be expended into

$$
\begin{gathered}
4 G / \pi a=\sigma_{32}^{\infty}\left(H_{11} \sigma_{32}^{\infty}+H_{12} D_{2}^{\infty}+H_{13} B_{2}^{\infty}\right)+ \\
+\Delta D_{2}^{\infty}\left(H_{21} \sigma_{32}^{\infty}+H_{22} D_{2}^{\infty}+H_{23} B_{2}^{\infty}\right)+\Delta B_{2}^{\infty}\left(H_{31} \sigma_{32}^{\infty}+H_{32} D_{2}^{\infty}+H_{33} B_{2}^{\infty}\right) .
\end{gathered}
$$


Below we present explicit expressions of $\mathrm{G}$ for some special cases by substituting Eqs. (61) and (62) into Eq. (92):

(i) If only $\sigma_{32}^{\infty}$ is applied, the result of (92) is

$$
G=\frac{\pi a}{4} H_{\sigma}\left(\sigma_{32}^{\infty}\right)^{2}
$$

where

$$
H_{\sigma}=H_{11}-\frac{H_{12}^{2} H_{33}\left(1+\lambda_{m}\right)-2 H_{12} H_{23} H_{31}+H_{13}^{2} H_{22}\left(1+\lambda_{e}\right)}{H_{22} H_{33}\left(1+\lambda_{e}\right)\left(1+\lambda_{m}\right)-H_{23}^{2}} .
$$

For $\lambda_{e}=\lambda_{m}=0$, Eq. (94) reduces to

$$
H_{\sigma}=H_{11}-\frac{H_{12}^{2} H_{33}-2 H_{12} H_{23} H_{31}+H_{13}^{2} H_{22}}{H_{22} H_{33}-H_{23}^{2}}=H_{11}^{*}(0) .
$$

In this case, Eq.(93) becomes (90).

(ii) If only $D_{2}^{\infty}$ is applied, we have from (92) that

$$
G=-\frac{\pi a}{4} H_{D}\left(D_{2}^{\infty}\right)^{2}
$$

where

$$
H_{D}=\frac{\lambda_{e} H_{22} H_{23}^{2}-\lambda_{e}\left(1+\lambda_{m}\right) H_{22}^{2} H_{33}}{H_{22} H_{33}\left(1+\lambda_{e}\right)\left(1+\lambda_{m}\right)-H_{23}^{2}} .
$$

(iii) If only $B_{2}^{\infty}$ is applied, we have

$$
G=-\frac{\pi a}{4} H_{B}\left(B_{2}^{\infty}\right)^{2}
$$

where 


$$
H_{B}=\frac{\lambda_{m} H_{33} H_{23}^{2}-\lambda_{m}\left(1+\lambda_{e}\right) H_{33}^{2} H_{22}}{H_{22} H_{33}\left(1+\lambda_{e}\right)\left(1+\lambda_{m}\right)-H_{23}^{2}} .
$$

\section{Numerical results}

Consider a $\mathrm{BaTiO}_{3}-\mathrm{CoFe}_{2} \mathrm{O}_{4}$ composite, where the matrix is the $\mathrm{CoFe}_{2} \mathrm{O}_{4}$ and the reinforced phase is the $\mathrm{BaTiO}_{3}$. Material constants of each phase can be found in the work of Li and Dunn (1998). Obviously, the effective properties of the composite depend on the volume fraction of its constituents. Many investigators, e.g., Li and Dunn (1998), Huang et al. (2000), Wu and Huang (2000), Li (2000), Aboudi (2001), Chen et al. (2002) and others, have investigated the effective properties of magneto-electro-elastic composites based on different approaches. Recently, Sih and Song (2003) presented some effective properties of the $\mathrm{BaTiO}_{3}-\mathrm{CoFe}_{2} \mathrm{O}_{4}$ composite for different volume fraction of $\mathrm{BaTiO}_{3}$. In the following numerical calculation, the used effective elastic and piezoelectric properties for a model piezoelectric/magnetic composite are selected as follows:

$$
\begin{array}{lll}
c_{44}=43 \times 10^{9} \mathrm{~Pa}, & e_{15}=11.6 \mathrm{C} / \mathrm{m}^{2}, & \kappa_{11}=11.2 \times 10^{-9} \mathrm{C}^{2} / \mathrm{Nm}^{2}, \\
d_{11}=0, & q_{15}=550 \mathrm{~N} / \mathrm{Am}, & \mu_{11}=590 \times 10^{-6} \mathrm{Ns}^{2} / \mathrm{C}^{2} .
\end{array}
$$

In addition, the dielectric permittivity and the magnetic permeability of air are respectively:

$$
\kappa_{0}=8.85 \times 10^{-12} \mathrm{C}^{2} / \mathrm{Nm}^{2}, \quad \quad \mu_{0}=1.26 \times 10^{-6} \mathrm{Ns}^{2} / \mathrm{C}^{2} .
$$

Numerical results are presented in Figs. 2-11, in which $\hat{G}=4 G / \pi a$. The units of $\sigma_{32}^{\infty}, D_{2}^{\infty}\left(D_{2}^{0}\right)$, $B_{2}^{\infty}\left(B_{2}^{0}\right)$ and $G$ are in $\mathrm{Pa}, \mathrm{C} / \mathrm{m}^{2}, \mathrm{~N} / \mathrm{Am}$ and $\mathrm{N} / \mathrm{m}$, respectively.

The numerical results for the selected model material are summarized as follows: 
(1) Figure 2 illustrates the electric field inside the crack as a function of $\lambda_{e}$ under a load of $\sigma_{32}^{\infty}=10^{5}, B_{2}^{\infty}=10^{-1}$, or $D_{2}^{\infty}=10^{-5}$ only, whereas Fig. 3 plots the magnetic field inside the crack as a function of $\lambda_{m}$ under a load of $\sigma_{32}^{\infty}=10^{4}, B_{2}^{\infty}=10^{-3}$, or $D_{2}^{\infty}=10^{-4}$ only. The results show that the signs of the electric field induced by the applied mechanical and magnetic loadings are opposite to that induced by the applied electric field, and the signs of the magnetic field induced by the applied mechanical and electric loadings are opposite to that induced by the applied magnetic field. When $\lambda_{e}=\lambda_{m}=0$, i.e., for permeable cracks, the electric (or magnetic) field inside the crack is independent of the applied magnetic (or electric) field. For an impermeable crack, $\lambda_{e} \rightarrow \infty$ and $\lambda_{m} \rightarrow \infty$, both the electric and magnetic fields approach zero.

(2) Figures 4-6 show the influence of crack permeability on the energy release rate under different loading conditions. Under purely mechanical loading, the energy release rate $\hat{G}$ is always positive (Fig.4). A permeable crack gives the highest value of $\hat{G}$, while an impermeable crack yields the lowest value of $\hat{G}$. If a purely electric or magnetic field is applied, the energy release rate is zero for a permeable crack, and negative for an impermeable or semi-permeable crack, as shown in Figs. 5 and 6. For a permeable crack under combined mechanical, electric and magnetic loading, the energy release rate is dependent on only the mechanical load, which is shown by the curve for $\lambda_{e}=0$ in Fig. 4 .

(3) Figures 7 and 8 show, respectively, the effects of the electric and magnetic fields on the energy release rate for an impermeable crack. For a given mechanical tension load, the 
electric/magnetic fields can either increase or decrease $\hat{G}$, which is dependent on the ratio of the applied electric/magnetic loading to the applied mechanical loading.

(4) Figures 9-11 give the energy release rate of a semi-permeable crack with a value of $\alpha=b / a=10^{-2}$. Under combined loading, the energy release rate can be positive or negative under an electric or magnetic load, depending on the value of the applied mechanical load, as shown in Fig. 9. For a given electric/magnetic loading, the mechanical load must reach a critical value before the energy release rate becomes positive. Obviously, the critical value of the mechanical load depends on the strengths of the electric/magnetic load. Figure 10 shows the energy release rate as a function of the applied electric load, $D_{2}^{\infty}$, with $B_{2}^{\infty}=0$ and $\sigma_{2}^{\infty}=0$, and $B_{2}^{\infty}=0$ and $\sigma_{2}^{\infty}=10^{6}$. In the absent of mechanical loading, both positive and negative electric loads lower the energy release rate. However, if a mechanical loading is applied also, a negative electric load decreases $\hat{G}$, but a positive electric load can either increase or decrease $\hat{G}$. This means that, for a fixed mechanical load, the effect of positive electric loading on the fracture behavior can be different from that of negative electric loading. Similarly conclusions can be drawn from Fig. 11 for the effects of magnetic loading. 


\section{Concluding Remarks}

Based on linear magneto-electro-elasticity, we study a crack in a magnetoelectroelastic solid under anti-plane deformation. To take the effect of the electric/magnetic fields inside the crack into account, the crack is modeled as a slender elliptical cavity containing air. Then, explicit expressions of the energy release rate are given for the elliptical flaw. Numerical results are also presented to show the variation of electromagneticelastic fields inside the crack and the values of the energy release rate with the flaw size. For a mathematical permeable crack with zero width, theoretical results show that the energy release rate is independent of the applied electric/magnetic loading. However, for a crack with a finite nonzero width, numerical results for a selected model material show that an applied magnetic field can induce a high electric field inside the crack and vise versa. In the absence of mechanical loading, an applied electric or magnetic load always tends to retard crack growth. However, in the presence of mechanical loading, an applied negative electric or magnetic load retards crack growth, while an applied positive electric or magnetic load can enhance or retard the growth. But, when the applied electric or magnetic load reaches a sufficiently high value, whether positive or negative, it always retards crack growth, even in the presence of mechanical load. In other words, the effect of electric/magnetic fields on the fracture behavior is mechanical load-dependent.

Finally, it should be noted that although the theoretical approach developed in the present study is for anti-plane deformation, it is very similar to the Stroh formalism of generalized 2D deformation. Therefore, we may call it the Stroh formalism for anti-plane deformation. The advantages of the Stroh formalism for anti-plane deformation lie in that it is much easy to be used and the obtained results can be readily checked by the conventional complex potential approach. Once one gets used to the Stroh formalism for anti-plane deformation, he/she can employ the Stroh formalism to explore general deformation problems without any difficulty. 


\section{Acknowledgments}

This work was supported by a grant from the Research Grants Council of the Hong Kong Special Administrative Region, China. TY Zhang thanks the Croucher Foundation for the Croucher Senior

Research Fellowship Award, which gave him more research time by releasing him from teaching duties. 


\section{References:}

Aboudi, J., 2001. Mircomechanical analysis of fully coupled electro-magneto-thermo-elastic multiphase composites. Smart Mater. Struct. 10, 867-877.

Chen, Z.R., Yu, S.W., Meng, L., Lin, Y., 2002. Effective properties of layered magneto-electroelastic composites. Composite Structures 57, 177-182.

Chung, M.Y., Ting, T.C.T., 1995. The Green function for a piezoelectric piezomagnetic anisotropic elastic medium with an elliptic hole or rigid inclusion. Philos. Mag. Lett. 72, 405-410.

Gao, C.F., Kessler, H., Balke, H., 2003. Crack problems in magnetoelectroelastic solids. Part I: exact solution of a crack. Int. J. Eng. Sci. 41, 969-981.

Gao, C.F., Kessler, H., Balke, H.,2003. Crack problems in magnetoelectroelastic solids. Part II: general solution of collinear cracks. Int. J. Eng. Sci.41, 983-994.

Huang, J. H., Liu, H.K., Dai, W.L., 2000. The optimized fiber volume fraction for magnetoelectric coupling effect in piezoelectric-piezomagnetic continuous fiber reinforced composites. Int. J. Eng. Sci.38, 1207-1217.

Pan, E., 2002. Three-dimensional Green's functions in anisotropic magneto-electro-elastic bimaterails. Z. angew. Math. Phys.53, 815-838.

Li, J.Y., Dunn, M.L., 1998. Micromechanics of magnetoelectroelastic composite materials: average field and effective behavior. J. Intell. Mater. Syst. Struct. 9, 404-416.

Li, J.Y.,2000. Magnetoelectroelastic multi-inclusion and inhomogeneity problems and their applications in composite materials. Int. J. Eng. Sci.38, 1993-2011.

Liu, J. X., Liu X.L., Zhao, Y.B., 2001. Green's functions for anisotropic magnetoelectro-elastic solids with an elliptical cavity or a crack. Int. J. Eng. Sci.39, 1405-1418. 
McMeeking, R.M., 1989. Electrostrictive forces near crack like flaws. J. Appl. Math. Phsy.40, 615627.

Sih, G.C., Song, Z.F., 2003. Magnetic and electric poling effects associated with crack growth in $\mathrm{BaTiO}_{3}-\mathrm{CoFe}_{2} \mathrm{O}_{4}$ composite. Theor. Appl. Fract. Mech. 39, 209-277.

Van Run, A.M.J.G., Terrell, D.R., Scholing, J.H., 1974. An in situ grown eutectic magnetoelectric composite material. J. Mater. Sci. 9,1710-1714.

Wang, B.L., Mai, Y.W., 2003. Crack tip field in piezoelectric/piezomagnetic media. Euro. J. Mech. A/Solids 22, 591-602.

Wang, X., Shen, Y.P., 2002. The general solution of three-dimensional problems in magnetoelectroelastic media. Int. J. of Eng. Sci. 40, 1069-1080.

Wu, T.L., Huang, J. H., 2000. Closed-form solutions for the magnetoelectric coupling coefficients in fibrous composites with piezoelectric and piezomagnetic phases. Int. J. Solids \& Structures 37, 2981-3009.

Zhang, T.Y., 1994. Effect of Sample Width on the Energy Release Rate and Electric Boundary Conditions along Crack Surfaces in Piezoelectric Materials, Inter. J. Fracture, 66, R33-R38.

Zhang, T.Y., Tong, P., 1996. Fracture Mechanics for a Mode III Crack in a Piezoelectric Material. Int. J. Solids Struct. 33, 343-359.

Zhang, T.Y., Qian, C.F., Tong, P., 1998. Linear electro-elastic analysis of a cavity or a crack in a piezoelectric material. Int. J. Solids Struct. 35, 2121-2149.

Zhang, T.Y., Zhao, M.H., Tong, P., 2002. Fracture of piezoelectric ceramics. Advances in Applied Mechanics 38, 147-289. 


\section{List of Figure Captions}

Fig.1. Anti-plane deformation of a magnetoelectroelastic solid with an elliptical cavity.

Fig.2. $D_{2}^{0}$ vs. $\lambda_{e}$ under the load of $\sigma_{32}^{\infty}=10^{5}, B_{2}^{\infty}=10^{-1}$, or $D_{2}^{\infty}=10^{-5}$ only.

Fig.3. $B_{2}^{0}$ vs. $\lambda_{m}$ under the load of $\sigma_{32}^{\infty}=10^{4}, B_{2}^{\infty}=10^{-3}$, or $D_{2}^{\infty}=10^{-4}$ only.

Fig.4. The effect of $\lambda_{e}$ on the energy release rate, $\hat{G}$, under purely mechanical loading.

Fig.5. The effect of $\lambda_{e}$ on the energy release rate, $\hat{G}$, under purely electrical loading.

Fig.6. The effect of $\lambda_{m}$ on the energy release rate, $\hat{G}$, under purely magnetic loading.

Fig.7. $\hat{G}$ for an impermeable crack under combined mechanical-electric loadings.

Fig.8. $\hat{G}$ for an impermeable crack under combined mechanical-magnetic loadings.

Fig.9. $\hat{G}$ vs. $\sigma_{32}^{\infty}$ under combined loading for a semi-permeable crack with the ratio $\alpha=b / a=10^{-2}$.

Fig.10. $\hat{G}$ vs. $D_{2}^{\infty}$ under combined loading for a semi-permeable crack with the ratio $\alpha=b / a=10^{-2}$.

Fig.11. $\hat{G} v s . B_{2}^{\infty}$ under combined loading for a semi-permeable crack with the ratio $\alpha=b / a=10^{-2}$. 
Fig.1. Anti-plane deformation of a magnetoelectroelastic solid with an elliptical cavity

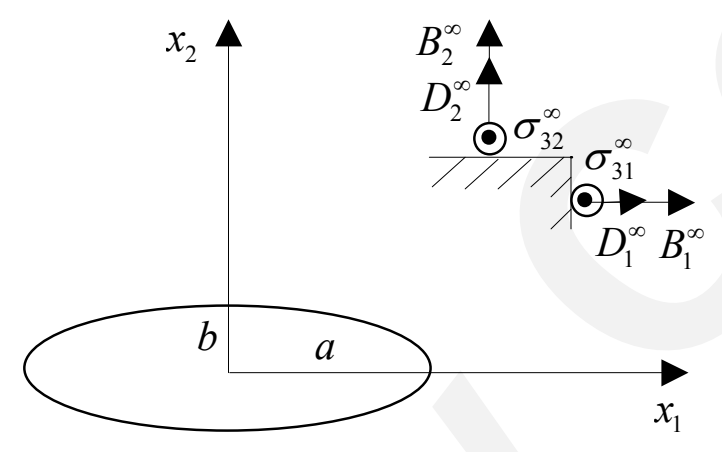


Fig.2. $D_{2}^{0} v s . \lambda_{e}$ under the load of $\sigma_{32}^{\infty}=10^{5}, B_{2}^{\infty}=10^{-1}$, or $D_{2}^{\infty}=10^{-5}$ only.

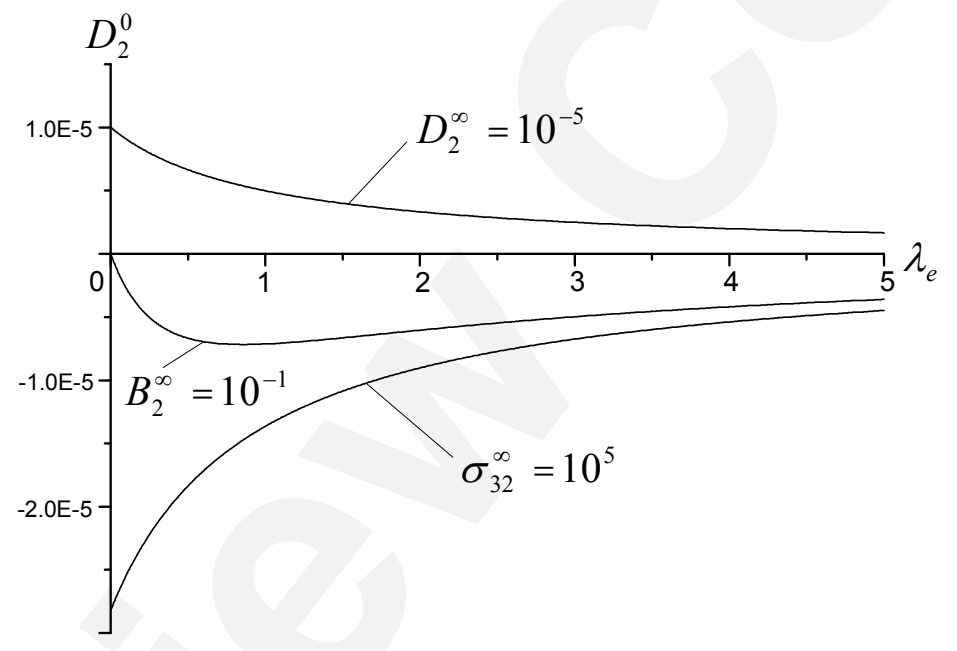


Fig.3. $B_{2}^{0}$ vs. $\lambda_{m}$ under the load of $\sigma_{32}^{\infty}=10^{4}, B_{2}^{\infty}=10^{-3}$, or $D_{2}^{\infty}=10^{-4}$ only.

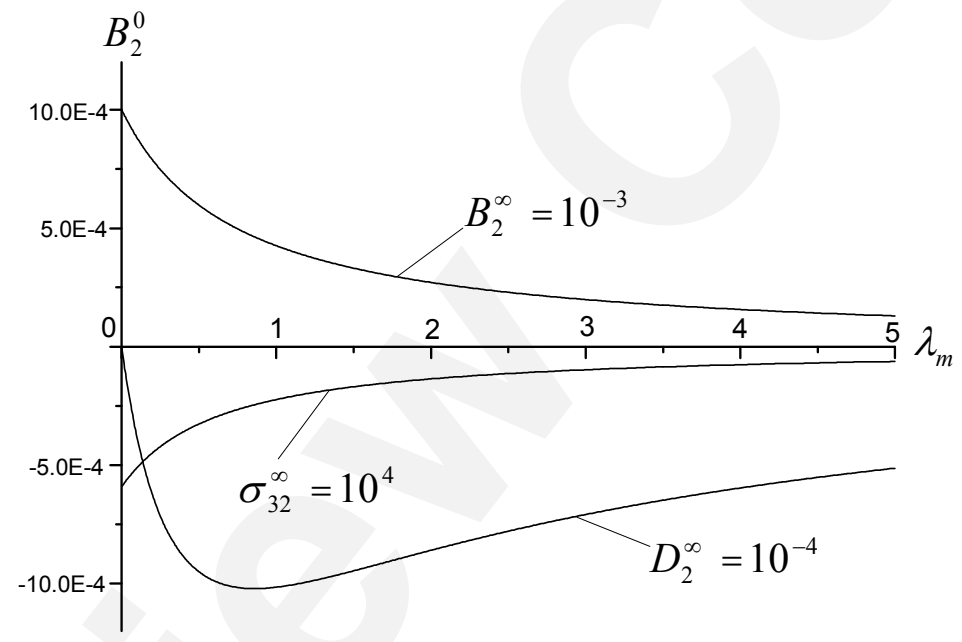


Fig.4. The effect of $\lambda_{e}$ on the energy release rate, $\hat{G}$, under purely mechanical loading.

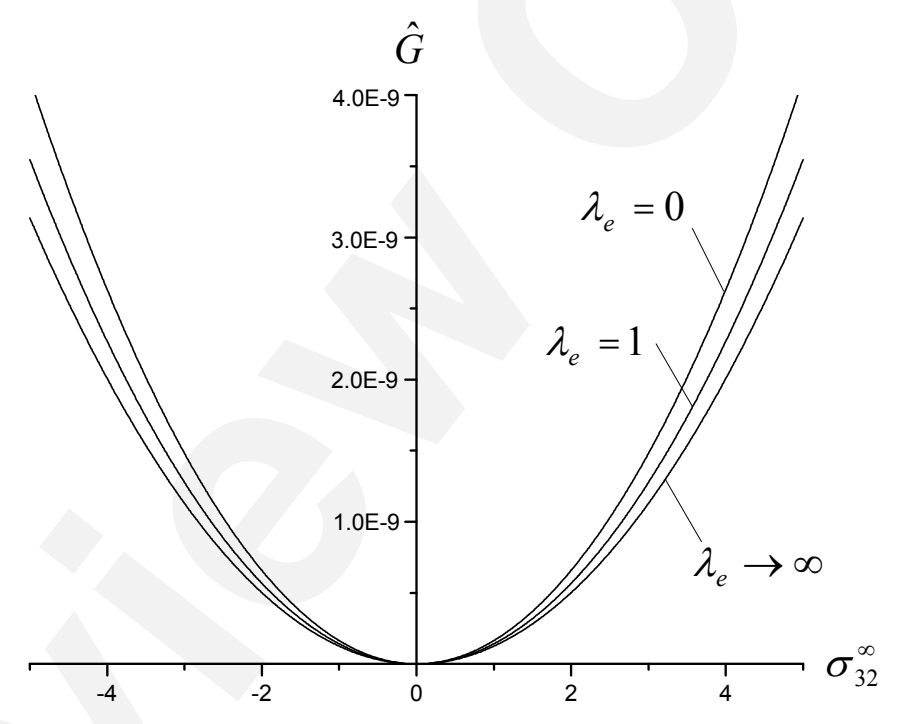


Fig.5. The effect of $\lambda_{e}$ on the energy release rate, $\hat{G}$, under purely electrical loading.

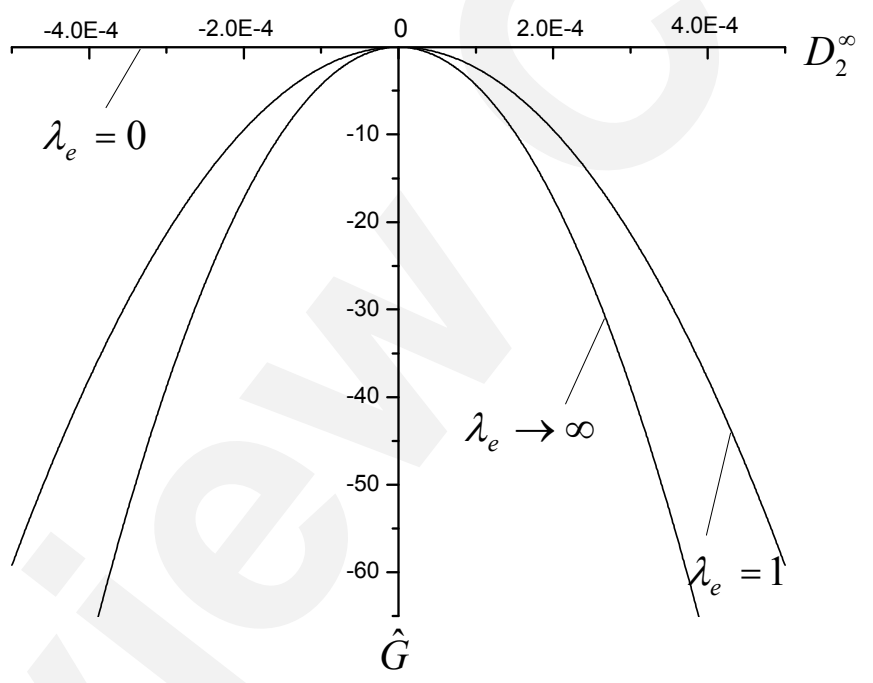


Fig.6. The effect of $\lambda_{m}$ on the energy release rate, $\hat{G}$, under purely magnetic loading.

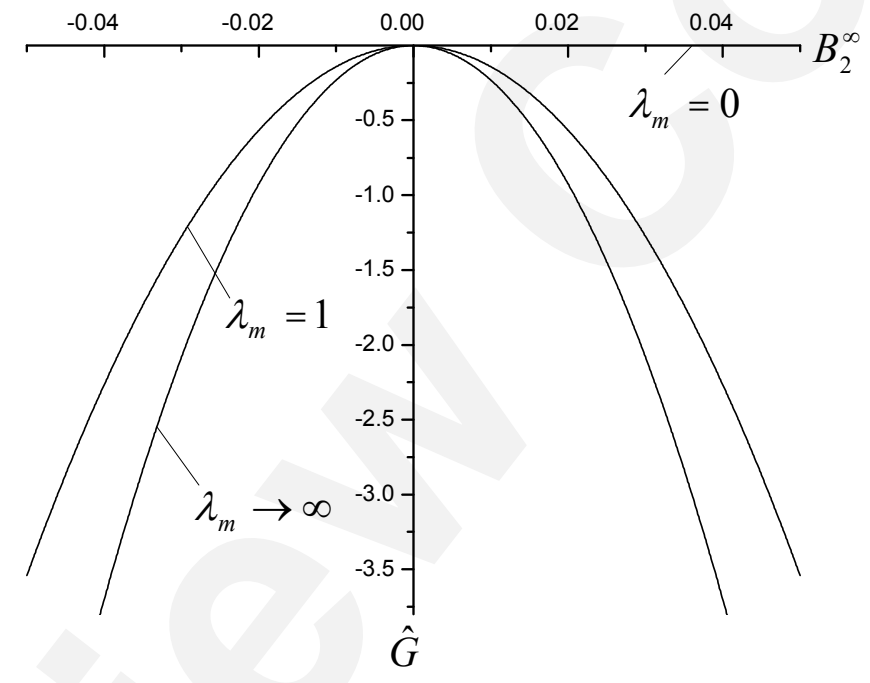


Fig.7. $\hat{G}$ for an impermeable crack under combined mechanical-electric loadings

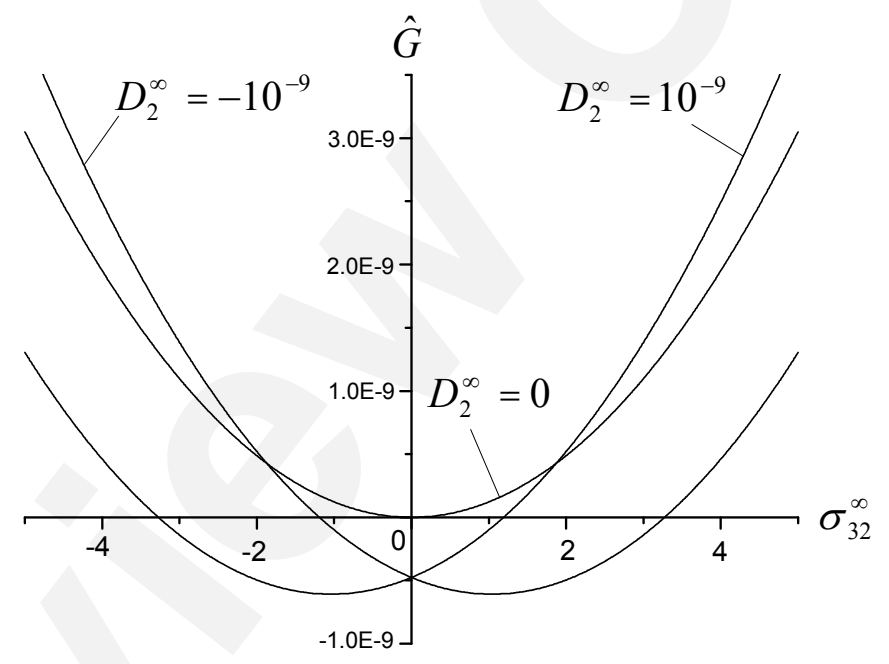


Fig.8. $\hat{G}$ for an impermeable crack under combined mechanical-magnetic loadings

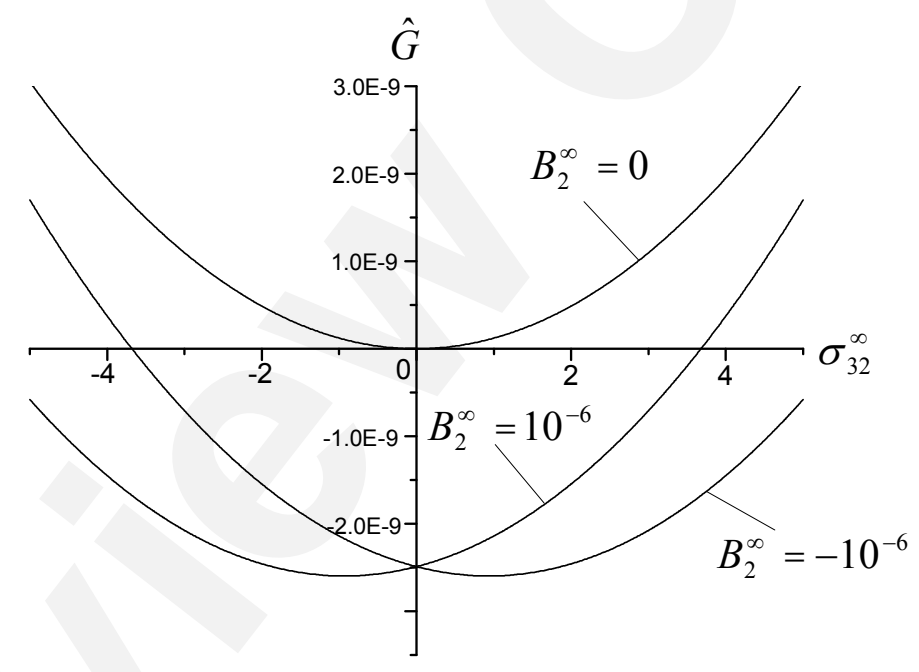


Fig.9. $\hat{G}$ vs. $\sigma_{32}^{\infty}$ under combined loading for a semi-permeable crack with the ratio

$$
\alpha=b / a=10^{-2} .
$$

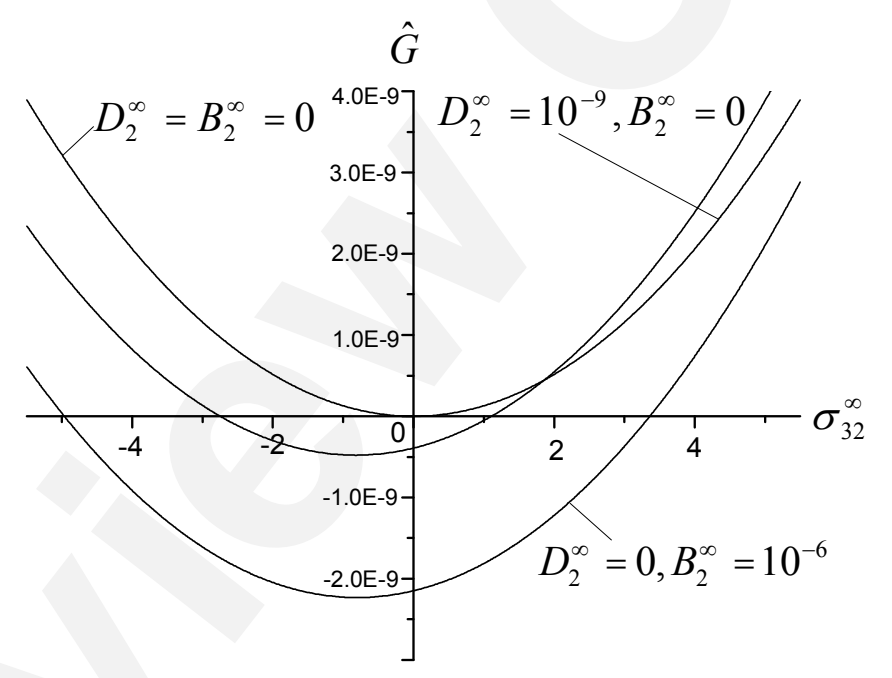


Fig.10. $\hat{G}$ vs. $D_{2}^{\infty}$ under combined loading for a semi-permeable crack with the ratio

$$
\alpha=b / a=10^{-2} .
$$

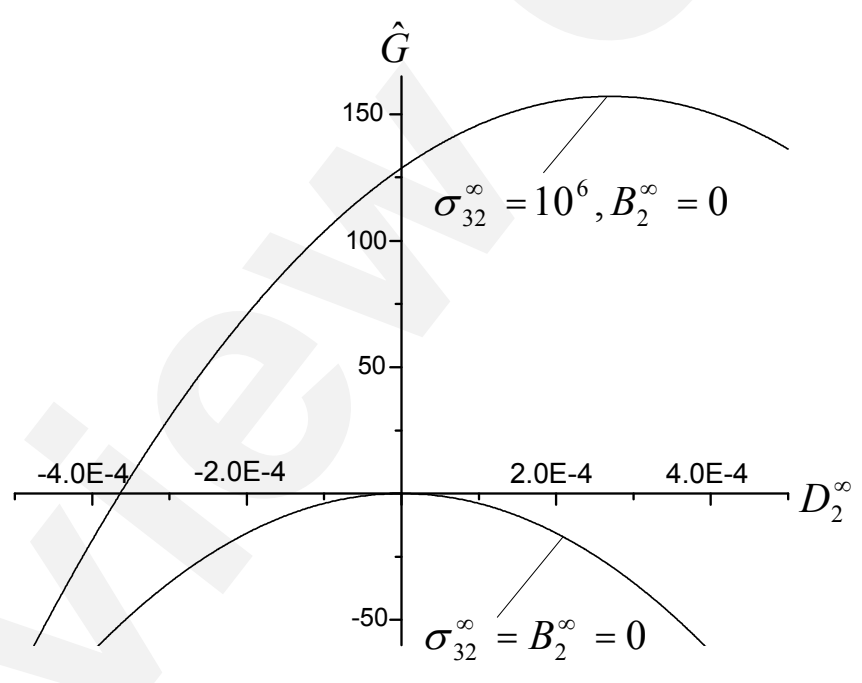


Fig.11. $\hat{G}$ vs. $B_{2}^{\infty}$ under combined loading for a semi-permeable crack with the ratio $\alpha=b / a=10^{-2}$.

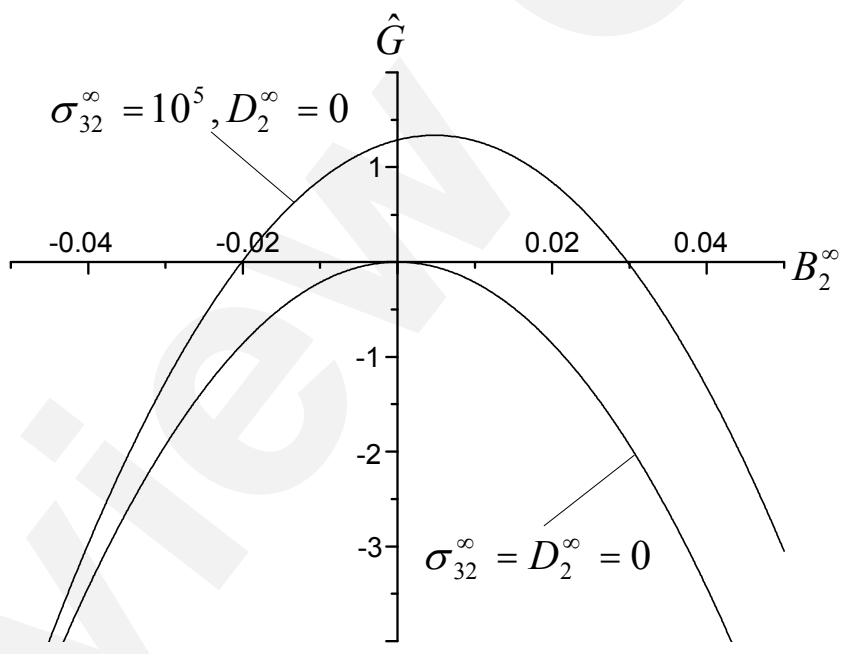

